\title{
High-resolution, high-aspect-ratio iridium-nickel composite nanoimprint molds
}

\author{
Chang-Sheng Lee, Yeong-Yuh Lee, and Karen S. L. Chong ${ }^{\text {a) }}$ \\ Institute of Materials Research and Engineering, Agency for Science Technology and Research (A*STAR), \\ 2 Fusionopolis Way, Innovis, \#08-03, Singapore 138634
}

Li Wang, Christian Dais, Francis Clube, and Harun H. Solak

Eulitha AG, Studacherstrasse 7b, 5416 Kirchdorf, Switzerland

Istvan Mohacsi and Christian David

Paul Scherrer Institut, 5232 Villigen PSI, Switzerland

Roger Bischofberger

microSWISS AG, Neugruet 45, LI-9496 Balzers, Liechtenstein

(Received 25 August 2016; accepted 28 October 2016; published 9 November 2016)

\begin{abstract}
Nanoimprint molds are traditionally made from silicon. Silicon molds suffer from distinct disadvantages as they are brittle, prone to damage, scratch easily, and can only be used in a planar format. This has limited their use in higher throughput systems where flexible molds are required such as in roll-to-roll and roll-to-plate systems. Nickel (Ni) molds, which are now de-rigueur in both batch and roller nanoimprint processes, can be used to address these problems, but fabrication and durability issues limit their availability and effectiveness in production. In this report, the authors introduce a fabrication route that has the potential to overcome the fabrication, quality, and wear problems of Ni molds. The new process relies on atomic layer deposition to form a smooth and highaspect ratio patterned layer of iridium (Ir) on a Ni substrate. A large area nanohole array mold was fabricated using displacement Talbot lithography to demonstrate this process. The authors show the use of such composite molds via a batch thermal imprinting process to fabricate $70 \mathrm{~nm}$ hole arrays onto polycarbonate templates with a $\pm 10 \%$ tolerance in diameter between the polycarbonate and the composite mold, and less than $1 \%$ of defects. (C) 2016 American Vacuum Society.
\end{abstract}

[http://dx.doi.org/10.1116/1.4967696]

\section{INTRODUCTION}

While nanoimprint lithography ${ }^{1,2}$ has surpassed expectations on resolution ${ }^{2,3}$ and throughput, ${ }^{4,5}$ there are still specific issues that need to be addressed to enable this lithographic process to be widely implemented for the fabrication of very high resolution features. Crucial to the nanoimprint process is the supply of a suitable mold that will allow an accurate and faithful replication of the features from the mold to the polymeric material. Such nanoimprint molds are typically made of silicon but can also be fabricated from other hard materials such as silicon oxide/ nitride, ${ }^{6}$ silicon carbide $(\mathrm{SiC}){ }^{7}$ and also quartz. ${ }^{8}$ However, there are inherent limitations to the use of such hard molds. They are often brittle and very expensive to fabricate especially when complex high resolution features are required, often not durable enough due to their susceptibility to scratches and cannot be applied in a roller process or a rollto-plate process. For silicon-type molds especially, their rigidity has often resulted in uneven imprints on rigid substrates. Solutions have been proposed to circumvent the use of hard molds and these often involve the use of soft molds fabricated either directly onto polymeric films or on resin coated films.

Imprint molds fabricated from polymers ${ }^{9-11}$ have been largely explored. While these soft molds offer some advantages

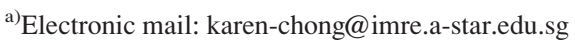

such as the ability to conform to uneven substrate surfaces ${ }^{12}$ and also can be applied onto roller processes, their properties are often dependent on the inherent properties of the polymer or resin from which they are fabricated. Soft molds directly fabricated onto polymer films such as ethylene(tetrafluoroethylene $)^{9}$ and even poly(dimethylsiloxane $)^{11}$ have been demonstrated though their uptake on the roller process is limited due to the higher processing conditions required on the soft molds and their poor resistance to solvents. The fabrication of soft molds has evolved to the use of UV curable resins ${ }^{13}$ such as siloxane based resins, epoxy based resins, and also polyhedral oligomeric silsesquioxane based resins to improve the properties of soft molds. Though successful, the creation of these soft molds is still reliant upon an additional coating of the specific resin on the supporting polymer substrate. Today, such molds are prized for their improved anti-stiction properties ${ }^{14-17}$ imparted from the properties of the resin.

Intuitively, the use of metal molds such as nickel molds has caught on in nanoimprinting. Such nickel molds are typically electroplated ${ }^{18}$ and are very durable and suitable for the roller process. While heavily utilized by most roller and batch processes today, there are still specific requirements on the performance of such nickel molds and this has led to other works by Chou et al. ${ }^{1,2,4}$ on the use of chemical vapor deposition processes to deposit thin amorphous silicon films onto polymer templates for "soft" silicon molds to recreate the flexibility of both a hard and soft mold in one process. 
Ni molds offer a number of advantages as described above, but fabrication of high-resolution and high-aspect ratio $\mathrm{Ni}$ molds presents significant challenges often limiting their use in many nanoimprinting applications. Typically, high-resolution patterns are created on a flat substrate like a silicon wafer by an e-beam or photolithography process. A suitable conductive seed film is then deposited on the patterned surface by a vacuum deposition process. This is followed by the electroplating process which forms a complementary copy of the patterned surface in a substantially thick (typically $0.5-5 \mathrm{~mm}$ ) $\mathrm{Ni}$ substrate. Finally, the formed $\mathrm{Ni}$ is released from the original substrate and used in imprint process after suitable cleaning and possible antiadhesion coating steps. As the pattern resolution and the aspect ratio of the desired structures increases, this process becomes less reliable. This is mainly due to the inability of the conventional seed layer deposition processes to form uniform conformal layers on such patterned surfaces, for example, bottom parts of deep holes or grooves are often not properly coated. Such inhomogeneity and voids in the seed layer prevent the faithful replication of the desired structure in the $\mathrm{Ni}$ electroplating process. In addition, $\mathrm{Ni}$ electroplating into such deep holes may lead to the formation of voids that compromise the strength and durability of the mold.

In this work, nanoimprint composite molds of nickel and iridium are fabricated to address these problems and to obtain high-fidelity nanostructures with the durability and lifespan of nickel molds. Specifically, we have used these molds to fabricate large-area $70 \mathrm{~nm}$ hole arrays on polycarbonate (PC) with less than $1 \%$ defects, voids, and other imperfections due to incomplete filling from the imprint area.

\section{EXPERIMENT}

\section{A. Production of masters with DUV displacement Talbot lithography}

The high-resolution masters required for producing the iridium-nickel molds were fabricated using displacement Talbot lithography (DTL). ${ }^{19}$ The exposures were performed on a PhableR 100 lithography tool which is specifically designed to perform DTL exposures. For the lithographic exposures, a deep ultraviolet (DUV) laser operating at a wavelength of $266 \mathrm{~nm}$ was used as the light source. ${ }^{20}$ Using the DTL method, an array of pillars with a diameter of $75 \mathrm{~nm}$ and period of $150 \mathrm{~nm}$ was first formed in a photoresist layer. This pattern was then used as an etch mask in a dry etching process to form Si pillars having a height of $90 \mathrm{~nm}$. An SEM image of the pattern etched in Si can be seen in Fig. 1.

\section{B. Production of iridium-nickel composite molds}

After dry etching, the silicon mold was coated with a $100 \mathrm{~nm}$ thick layer of iridium using plasma enhanced atomic layer deposition in a Picosun R200 ALD system equipped with an inductively coupled plasma source. The process consisted of 2840 cycles of exposure to a precursor of $\operatorname{Ir}(\mathrm{acac})_{3}$ ( $\mathrm{acac}=2,4$-pentadionato) and to a $\mathrm{O}_{2}$ plasma as reactant. The reactor chamber temperature was set to $370^{\circ} \mathrm{C}$. The gas

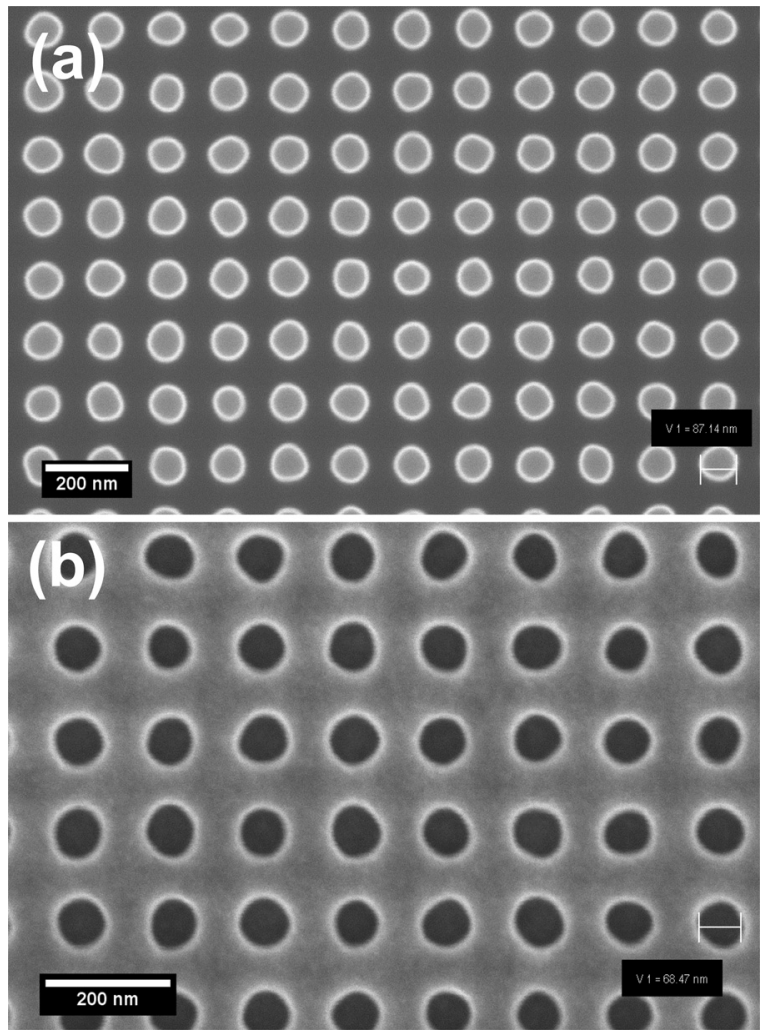

FIG. 1. (a) SEM images of (a) the pattern etched in Si. (b) The complementary pattern on the composite iridium-nickel mold. In both images, the period (center-to-center distance between features is $150 \mathrm{~nm}$.

flow of the plasma source was $50 \mathrm{sccm}$, and the power of the plasma unit was set to $2 \mathrm{~kW}$. Subsequently, a $0.5 \mathrm{~mm}$ thick $\mathrm{Ni}$ layer was electroplated on the Ir layer, and the resulting iridium-nickel composite mold was detached from the $\mathrm{Si}$ substrate. The process flow is illustrated in Fig. 2. The patterned $\mathrm{Si}$ wafer was used directly after the last reactive ion etching step without any additional surface preparation steps. In our experiment the Ir/Ni hybrid mold detached readily from the Si substrate. No damage or degradation of the mold surface due to the detachment process was observed on the mold.

\section{Nanoimprint process using composite molds}

The free-standing PC films (Young Chang High Tech Materials Co., Ltd., Republic of South Korea) used in this study are about $184 \mu \mathrm{m}$ thick and have a glass transition temperature of $150{ }^{\circ} \mathrm{C}$. There are various free standing polymers film such as PC and poly-(methyl methacrylate) (PMMA) that we can imprint on. Aside from structural differences in polymer chain, PC and PMMA also have very close glass transition temperatures 125 and $100^{\circ} \mathrm{C}$, respectively; therefore, there should be no challenges to imprint PMMA. The surface energy for PC is lower than that of PMMA; this can contribute to a slightly easier demolding process for PC than PMMA. $^{21,22}$ PC was used throughout the experiment to achieve consistency for that material. The composite mold was first precoated with an antistiction coating, perfluorodecyltrichlorosilane, via a vapor deposition process. The 


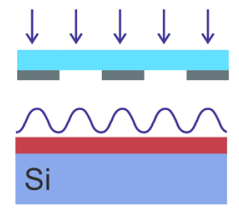

(a)

(d)

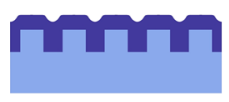

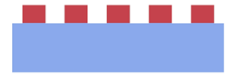

(b)

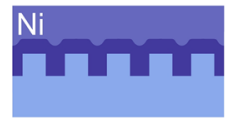

(e)

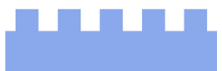

(c)

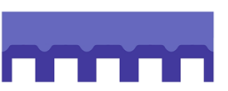

(f)
FIG. 2. (Color online) Schematic diagram of the process flow for the fabrication of the iridium-nickel composite mold. (a) DTL exposure of photoresist coated on Si (b) pattern developed in photoresist (c) etched pattern in Si. (d) ALD deposition of Ir layer. (e) Ni electroplating. (f) Iridium-nickel mold after releasing from the Si master.

composite mold area was $40 \times 40 \mathrm{~mm}$ with a uniform square array of $70 \mathrm{~nm}$ diameter pillars.

A thermal nanoimprint lithography process was carried out in this study. One cycle of the imprint process comprises two main steps and was carried out in an Obducat Nanoimprinter; first, the temperature was elevated to $160^{\circ} \mathrm{C}$ $\left(10^{\circ} \mathrm{C}\right.$ above the glass transition temperature of the imprint material), and the composite mold was embossed into the material at a pressure of 40 bars for $300 \mathrm{~s}$. Then, the system was cooled to $25^{\circ} \mathrm{C}$ before the imprinted material, and mold were separated in the demolding step. Significant force is required to demold the film from the composite mold, but the film was not damaged or deformed during the process from the first to 100th imprint. In this study, the composite mold was subjected to 100 imprint cycles.

\section{Surface characterization of mold and nanoimprinted films}

Composite mold durability, lifespan, and the imprint quality are the most important factors in this experiment. The composite mold is subjected to repeated processing at high temperature of $160^{\circ} \mathrm{C}$ and then cooling to room temperature at $25^{\circ} \mathrm{C}$. The composite mold was used for a total of 100 imprint cycles without an additional antistiction coating process between cycles. The criteria defined for assessing the polymer films fabricated in the previous step are described in the supplementary material under Table S1. ${ }^{23}$ The fabricated films were assessed for macroscopic defects such as residue stains, microscopic defects such as cracks and scratches, and also the ease of demolding. The patterns were imaged by atomic force microscopy (Bruker Dimension ICON) under tapping mode (AFM tip Model: NCHV, Bruker Probes) for height analysis and scanning electron microscope (JEOL FESEM 6700) for surface analysis. Each polymer film at 0th, 30th, 60th, and 100th imprint was divided into nine sections, and three SEM images of each section are collected-a total of 27 images were subsequently acquired for each film (refer to supplementary material Figs. S1 and $\mathrm{S} 2^{23}$ ).

\section{RESULTS AND DISCUSSION}

\section{A. Characterization of composite mold}

An SEM image of the resulting composite mold is shown in Fig. 1(b). From our SEM observations, the holes on the mold are fully formed, and no evidence of voids or other imperfections due to incomplete filling of the Si master by the seed layer are noticeable. In contrast, conventional sputtering and physical vapor deposition processes do not yield such smooth and faithful replication at this high resolution and significant aspect ratio.

Instead of the nickel surface in a conventional nickel mold, the exposed surface of the iridium-nickel composite mold is iridium. Iridium, being harder than nickel, is potentially more robust and durable. Visual inspections of the iridium-nickel composite mold after each imprint cycle did not show any noticeable cracks or delamination of the patterned area. This shows that the interface between the iridium outermost layer and underlying nickel base is durable and is able to withstand the thermal imprint environment used in this study. AFM measurements on the composite mold were carried out for a comparison between preimprint composite mold feature and postimprint mold (refer to supplementary material Figs. S3 and $\mathrm{S} 4{ }^{23}$ ). The AFM measurements indicate that the composite mold had maintained its pattern fidelity (70 $\mathrm{nm}$ holes array) after 30 imprints and did not show noticeable microcracks on the mold surface.

After the 100th imprint cycle, the surfaces of the composite mold were further visualized using SEM to assess and check for any forms of physical damage to the mold. Representative SEM images are shown in Fig. 3. From our surface scans, no observable physical damage was found. Thus, the composite mold showed excellent durability over 100 imprint cycles.

\section{B. Characterization of nanoimprinted PC film}

Figure 4 shows the SEM images of the imprinted polycarbonate templates from the first imprint and 100th batch thermal imprint cycles. To determine the consistency of the imprints quality and the fidelity of pattern transfer with the repeated use of the composite mold, image processing software IMAGEJ was used to measure the diameters of the nanopillars on the composite mold and PC templates at 30th, 60th, 90th, and 100th imprint cycle. Diameter measurements on the SEM images of the composite mold indicate that the average diameter of the nanostructures was $80 \pm 2 \mathrm{~nm}$, while the average diameter of the nanopillars on the PC templates ranges from 75 to $78 \mathrm{~nm}$ at various imprint cycles [refer to Fig. 5(a)]. The maximum difference between the measured diameter of the nanostructures on the composite mold and the nanopillars on the PC templates is less than $10 \%$ $(6.25 \%)$. Though there are small differences in the measured diameters, statistical analysis over the diameters of the nanopillars did not show any statistical differences.

In this study, the number of defects was enumerated by counting the number of missing pillar nanostructures from scanning electron micrographs of the imprinted PC film taken at a magnification of $10000 \times$. At this magnification, 

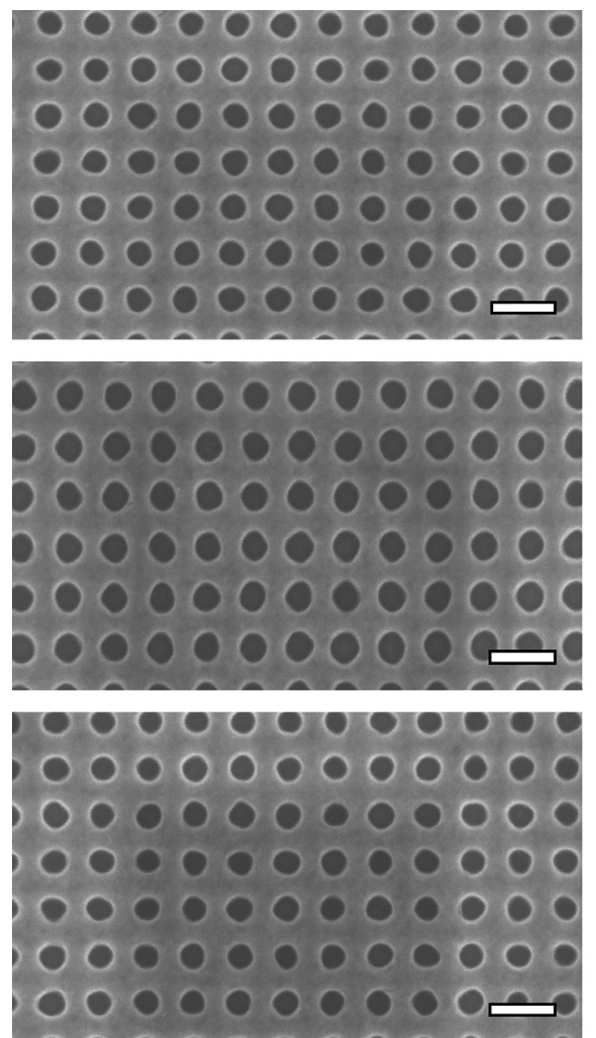
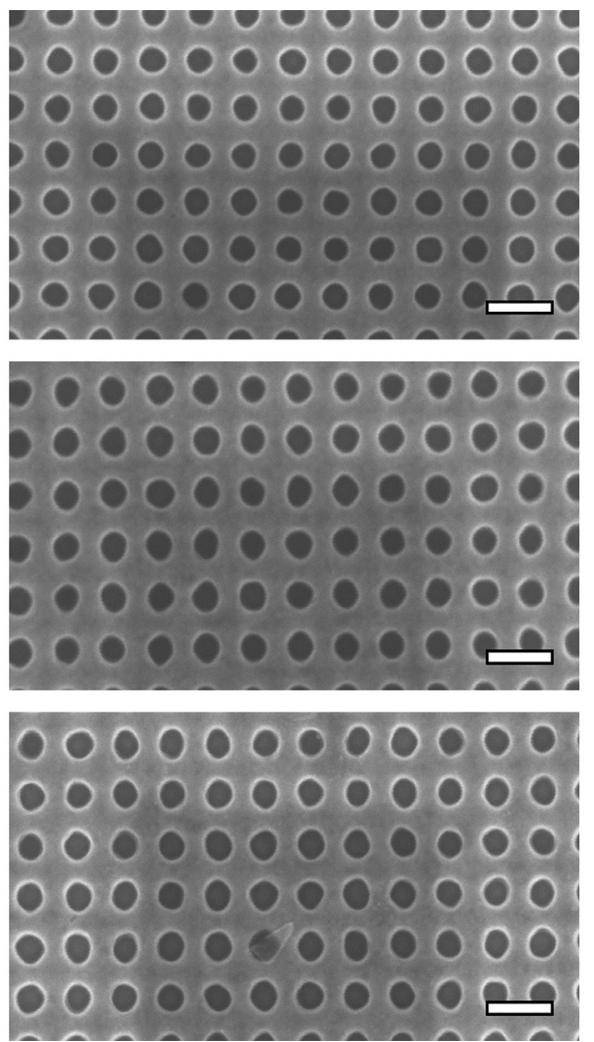
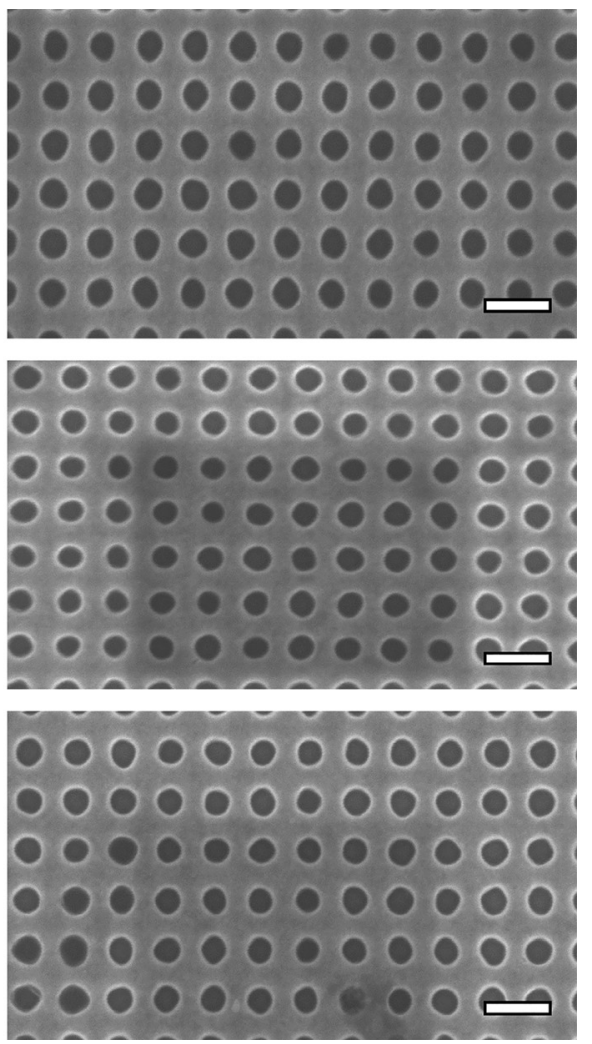

FIG. 3. SEM images of the composite mold at each of the nine sections after 100th imprint cycles showing no observable physical damage to the mold surface. The scale bar represents $200 \mathrm{~nm}$ for all figures shown.

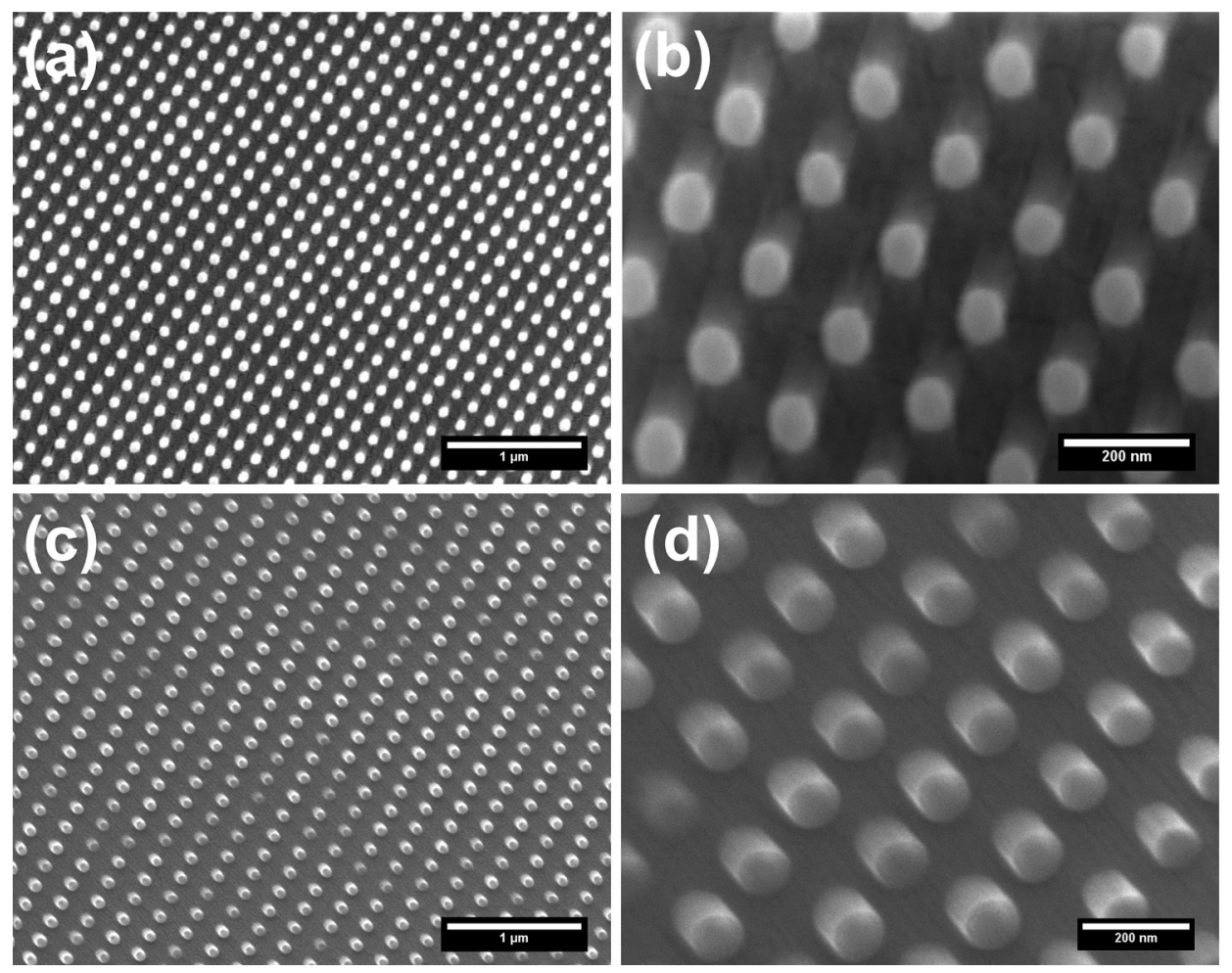

FIG. 4. SEM images of (a) imprinted polycarbonate template from the first imprint, (b) the imprinted polycarbonate from the first imprint at high magnification, (c) imprinted polycarbonate templates from the 100th imprint, and (d) the imprinted polycarbonate template from the 100th imprint at high magnification. 

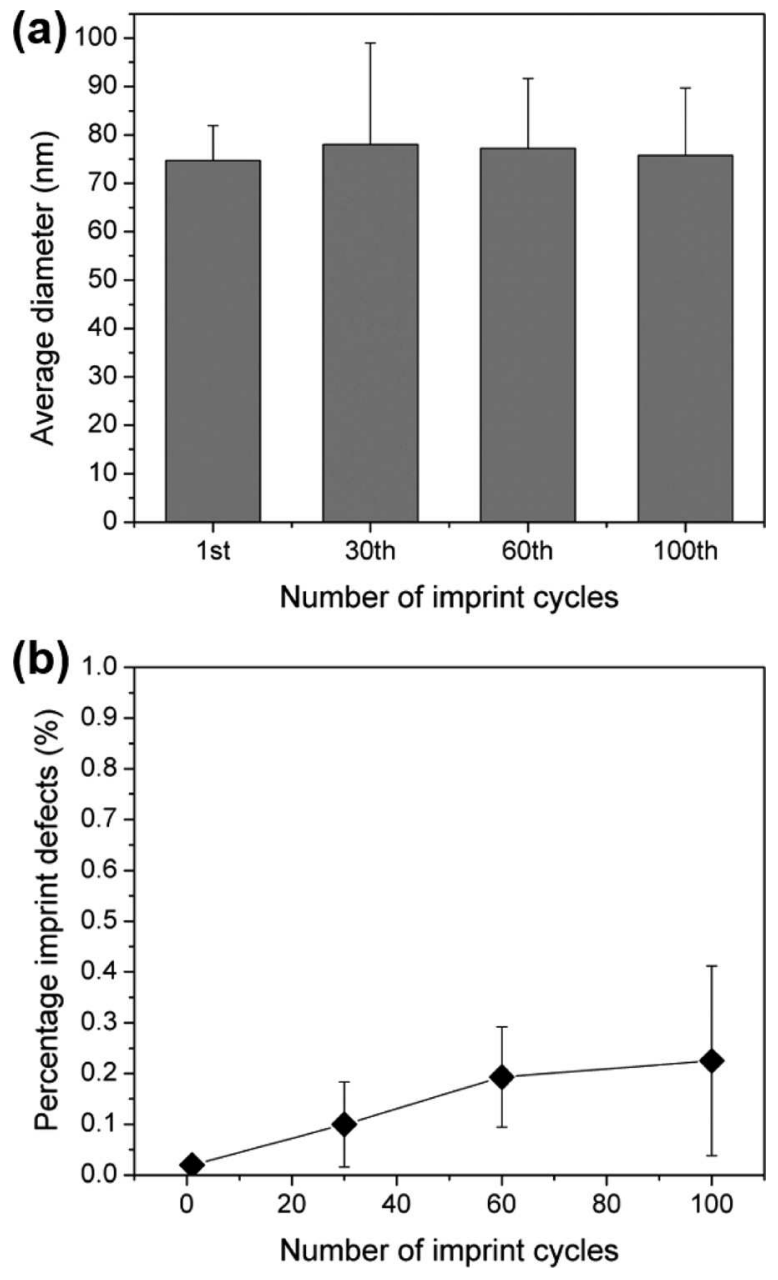

FIG. 5. (a) Average diameter of imprinted nanostructures measured from SEM images. (b) Average percentage imprint defects over 100 cycles of thermal imprinting steps.

there are at least 4312 nanopillars, and the area of the image is approximately $119 \mu \mathrm{m}^{2}$ within a frame (the total area of approximately $87040 \mu \mathrm{m}^{2}$ in one imprint). By sampling 27 fields of view on the PC templates over different sections of the imprinted surface, the percentage defects were enumerated and reported in Fig. 5(b). Although the defects increase after more imprint cycles, the defect percentage is measured to be within less than $1 \%$ of the total area. The increase in defect is not likely due to the mold damage but rather due to the wear-down of the antistiction layer on the surface of the mold.

\section{SUMMARY AND CONCLUSIONS}

To circumvent the technical challenges surrounding the fabrication of conventional nanopatterned nickel molds, iridium-nickel composite molds have been fabricated by first introducing an iridium seed layer on the silicon master by atomic layer deposition (ALD) before electroplating the mold with nickel. Here, we demonstrated that the highresolution composite molds thus fabricated have highfidelity; excellent durability over 100 imprint cycles and produce quality imprints of $70 \mathrm{~nm}$ pillar arrays onto the polycarbonate films with a $\pm 10 \%$ tolerance in diameter between the polycarbonate and the composite. Therefore, such iridium-nickel composite molds are viable flexible nanoimprint molds for the mass-production of large-area sub-100 nm nanostructures with excellent durability and lifespan.

\section{ACKNOWLEDGMENT}

The authors acknowledge the A*STAR Nanoimprint Foundry for funding support (under Project Code No. 1325307091).

${ }^{1}$ S. Y. Chou, P. R. Krauss, and P. J. Renstrom, Appl. Phys. Lett. 67, 3114 (1995).

${ }^{2}$ S. Y. Chou, J. Vac. Sci. Technol., B 14, 4129 (1996).

${ }^{3}$ M. Häffner, A. Heeren, M. Fleischer, D. P. Kern, G. Schmidt, and L. W. Molenkamp, Microelectron. Eng. 84, 937 (2007).

${ }^{4}$ S. Y. Chou and P. R. Krauss, Microelectron. Eng. 35, 237 (1997).

${ }^{5}$ A. Lebib, Y. Chen, J. Bourneix, F. Carcenac, E. Cambril, L. Couraud, and H. Launois, Microelectron. Eng. 46, 319 (1999).

${ }^{6}$ Y. Zhao, E. Berenschot, M. de Boer, H. Jansen, N. Tas, J. Huskens, and M. Elwenspoek, J. Micromech. Microeng. 18, 064013 (2008).

${ }^{7}$ L. Heon and J. Gun-Young, U.S. patent 6,916,511 B2 (12 July 2005).

${ }^{8}$ D. G. Choi, J. H. Jeong, Y. S. Sim, E. S. Lee, W. S. Kim, and B. S. Bae, Langmuir 21, 9390 (2005).

${ }^{9}$ D. R. Barbero, M. S. M. Saifullah, P. Hoffmann, H. J. Mathieu, D. Anderson, G. A. C. Jones, M. E. Welland, and U. Steiner, Adv. Funct. Mater. 17, 2419 (2007).

${ }^{10}$ K. Pfeiffer, G. Bleidiessel, G. Gruetzner, H. Schulz, T. Hoffmann, H. C. Scheer, C. M. Sotomayor Torres, and J. Ahopelto, Microelectron. Eng. 46, 431 (1999).

${ }^{11}$ Z. Li et al., Nano Lett. 9, 2306 (2009).

${ }^{12}$ J.-H. Chang, F.-S. Cheng, C.-C. Chao, Y.-C. Weng, S.-Y. Yang, and L. A. Wang, J. Vac. Sci. Technol., A 23, 1687 (2005).

${ }^{13}$ S. J. Choi, P. J. Yoo, S. J. Baek, T. W. Kim, and H. H. Lee, J. Am. Chem. Soc. 126, 7744 (2004).

${ }^{14}$ J. Moresco, C. H. Clausen, and W. Svendsen, Sens. Actuators, B 145, 698 (2010).

${ }^{15}$ C. W. Wu, Y. K. Shen, S. Y. Chuang, and C. S. Wei, Sens. Actuators, A 139, 145 (2007).

${ }^{16}$ S. Park, H. Schift, C. Padeste, B. Schnyder, R. Ü. Kötz, and J. Gobrecht, Microelectron. Eng. 73-74, 196 (2004).

${ }^{17}$ H. Sun, J. Liu, P. Gu, and D. Chen, Appl. Surf. Sci. 254, 2955 (2008).

${ }^{18}$ R. Ruprecht et al., Microsyst. Technol. 4, 28 (1997).

${ }^{19}$ H. H. Solak, C. Dais, and F. Clube, Opt. Express 19, 10686 (2011).

${ }^{20}$ L. Wang, F. Clube, C. Dais, H. H. Solak, and J. Gobrecht, Microelectron. Eng. 161, 104 (2016).

${ }^{21}$ L. Peng, Y. Deng, P. Yi, and X. Lai, J. Micromech. Microeng. 24, 13001 (2014).

${ }^{22}$ M. E. Dirckx and D. E. Hardt, J. Micromech. Microeng. 21, 085024 (2011).

${ }^{23}$ See supplementary material at http://dx.doi.org/10.1116/1.4967696 for more characterization of hybrid mold and imprinted films. 